\title{
The crystal structure of a compartmental heptadentate ligand
}

\author{
$\underline{\text { Matilde Fondo }}^{\mathrm{a}}$, Noelia Ocampo ${ }^{\mathrm{a}}$, Ana M. García-Deibe, ${ }^{\mathrm{a}}$ Jesús Sanmartín ${ }^{\mathrm{b}}$ \\ a Departamento de Química Inorgánica, Facultade de Ciencias, Universidade de Santiago de \\ Compostela, E-27002 Lugo, Spain. E-mail: matilde.fondo@usc.es \\ b Departamento de Química Inorgánica, Facultade de Química, Universidade de Santiago de \\ Compostela, E-15782 Santiago de Compostela, Spain
}

\begin{abstract}
2-(2-hydroxyphenyl)-1,3-bis[4-(2-hydroxyphenyl)-3-azabut-3-enyl]-1,3-imidazolidine $\left(\mathrm{H}_{3} \mathrm{~L}\right)$ was obtained with high purity by Schiff's condensation between 5-bromosalicyaldehyde and triethylenetetramine. Its recrystallisation in methanol yields single crystals suitable for X-ray diffraction studies. $\mathrm{H}_{3} \mathrm{~L}$ crystallises in the monoclinic system, space group $P 2_{1} / \mathrm{c}$, with $a=21.641(3) \AA, b=11.1217(14) \AA, c=11.4156(15) \AA$ and $\beta=90.016^{\circ}$.
\end{abstract}

\section{Introduction}

The design and synthesis of multidentate ligands is an essential step in the search for new polynuclear complexes of predefined architectures, an area of growing significance in the field of molecular materials. Following with our work in this topic, we describe herein the crystal structure of the potential heptadentate compartmental donor $\mathrm{H}_{3} \mathrm{~L}$.

\section{Results and discussion}

A perspective view of the molecule is shown in Fig. 1. All the angles and distances (Table 1) are in the range of those expected for this kind of Schiff base and do not deserve further consideration [1]. The crystal data show that the central phenol oxygen atom is disordered over two sites (O103 and O13') at 0.5 partial occupancies.

The ligand adopts a conformation which is determined by the formation of a five-membered imidazolidine ring and by the presence of strong intramolecular hydrogen bonds between the phenol functions and the nitrogen atoms. Accordingly, the terminal phenol oxygen atoms interact with the imine nitrogen atoms (distance O101 $\cdots \mathrm{N} 101=2.607(4) \AA$ and O102 $\cdots$ N102 $=2.598(4) \AA$ ). Besides, the disordered central phenol function is hydrogen bonded to both imidazolidine nitrogen atoms (distance O103 $\cdots \mathrm{N} 102=2.790(6) \AA$ and

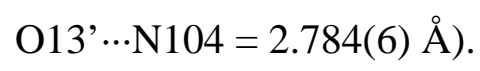




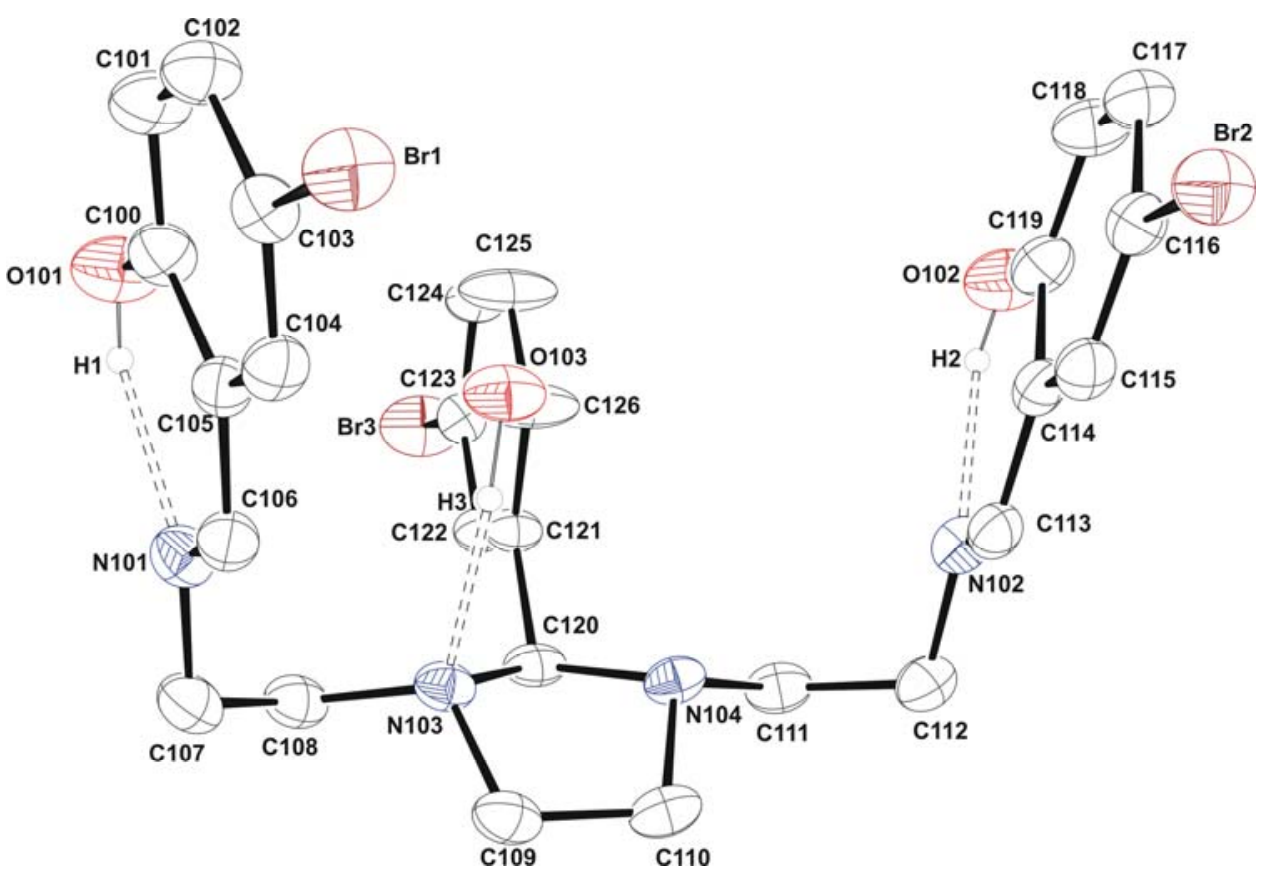

Fig.1

Table 1. Selected distances $(\AA)$ and angles $\left({ }^{\circ}\right)$ for $\mathrm{H}_{3} \mathrm{~L}$

\begin{tabular}{llll}
\hline O101-C100 & $1.343(4)$ & O101-C100-C101 & $118.9(3)$ \\
C103-Br1 & $1.900(3)$ & O101-C100-C105 & $121.3(3)$ \\
N101-C106 & $1.274(4)$ & C104-C103-Br1 & $119.9(3)$ \\
N101-C107 & $1.454(5)$ & C102-C103-Br1 & $119.1(3)$ \\
C108-N103 & $1.454(4)$ & C106-N101-C107 & $117.9(3)$ \\
N103-C120 & $1.464(4)$ & C108-N103-C120 & $113.3(3)$ \\
N103-C109 & $1.476(4)$ & C108-N103-C109 & $113.8(3)$ \\
C110-N104 & $1.476(4)$ & C120-N103-C109 & $103.5(3)$ \\
N104-C111 & $1.455(4)$ & C111-N104-C120 & $113.2(3)$ \\
N104-C120 & $1.467(4)$ & C111-N104-C110 & $114.1(3)$ \\
N102-C113 & $1.273(4)$ & C120-N104-C110 & $103.5(3)$ \\
C116-Br2 & $1.903(3)$ & C113-N102-C112 & $118.2(3)$ \\
C119-O102 & $1.340(4)$ & C115-C116-Br2 & $120.0(3)$ \\
C123-Br3 & $1.888(3)$ & C117-C116-Br2 & $118.7(3)$ \\
C126-O103 & $1.463(7)$ & O102-C119-C118 & $119.4(3)$ \\
C126-O13' & $1.474(7)$ & O102-C119-C114 & $121.4(4)$ \\
O101-C100 & $1.343(4)$ & N103-C120-N104 & $100.5(2)$ \\
C103-Br1 & $1.900(3)$ & C124-C123-Br3 & $118.6(2)$ \\
C106-N101 & $1.274(4)$ & C122-C123-Br3 & $120.6(2)$ \\
N101-C107 & $1.454(5)$ & C121-C126-O103 & $117.8(4)$ \\
N103-C120 & $1.464(4)$ & C125-C126-O103 & $116.1(4)$ \\
N103-C109 & $1.476(4)$ & C121-C126-O13' & $117.2(4)$ \\
& & C125-C126-O13 & $116.1(4)$ \\
\hline
\end{tabular}

\section{Experimental}

$\mathrm{H}_{4} \mathrm{~L}$ was isolated as follows: 5-bromo-salicylaldehyde $(0.87 \mathrm{~g}, 4.32 \mathrm{mmol})$ was added to a methanol $(10 \mathrm{~mL})$ solution of triethylentetramine $(0.21 \mathrm{~g}, 1.44 \mathrm{mmol})$. The solution was stirred in air for $4 \mathrm{~h}$. and a yellow solid precipitated, being subsequently filtered off. (0.800 g, $80 \%), \mathrm{mp}=156-158{ }^{\circ} \mathrm{C}$.

\section{References}

[1] L-W. Yang, S. Liu, E. Wong, S. J. Rettig, C. Orvig, Inorg. Chem. 1995, 34, 2164. 